\title{
Non-degenerate Developable Triangular Bézier Patches
}

\author{
Alicia Cantón and Leonardo Fernández-Jambrina
}

\begin{abstract}
In this talk we show a construction for characterising developable surfaces in the form of Bézier triangular patches. It is shown that constructions used for rectangular patches are not useful, since they provide degenerate triangular patches. Explicit constructions of nondegenerate developable triangular patches are provided.
\end{abstract}

\section{Introduction}

It is well known that developable surfaces play an important role in design in several branches of industry, such as naval and textile. Even architectural structures have been designed using developable surfaces. In these industries surfaces are designed which mimic properties of the materials that are used in production, which are intended to be deformed from plane sheets of metal or cloth just by folding, cutting or rolling, but not stretching. This sort of industrial procedures are less expensive or do not alter the properties of the material and therefore developable surfaces are favoured.

In spite of their importance, developable surfaces are not easy to design within the standard framework of NURBS surfaces. The null gaussian curvature condition is a cubic expression in the parametrization of the surface and can be solved analitically just for low degrees.

This does not mean that NURBS developable surfaces have not been used in design. On the contrary, pieces of plane, cylinders and cones have been used extensively. However, the general case of developable surfaces $[1,2]$, tangent surfaces, has not received the same attention, though it is by large the most important case of developable surfaces.

Since the seminal papers by Mancewicz and Frey [3], Frey and Bindschadler [4] at General Motors, several approaches have been used to cope with developable surfaces:

- Solving null curvature equations for low degrees: papers by Aumann [5], Lang and Röschel, [6], Chalfant and Maekawa [7]. 
- Projective geometry methods: planes and points are exchanged using duality: Bodduluri and Ravani [8], Pottmann and Farin [9], Pottmann and Wallner [10].

- Based on the de Casteljau algorithm: Chu and Séquin [11], Aumann [12], [13] and Fernández-Jambrina [14].

This last approach has been profitable for obtaining results with tensor product patches of developable surfaces and in this paper we would like to derive an extension to triangular patches.

The paper is organised as follows. Section 2 is devoted to ruled triangular patches. Section 3 provides a quick overview of differential geometry of developable surfaces. Section 4 reviews construction of tensor product developable surfaces. This approach is extended to triangular patches in Section 5. Finally, cylindrical and conical triangular patches are described in Section 6 .

\section{Ruled Triangular Bézier Patches}

Triangular Bézier patches are an alternative to tensor product patches for designing polynomial surfaces. Instead of dealing with parametrizations of degree $n_{1}$ in a variable and degree $n_{2}$ in the other one, triangles are parametrizations of overall degree $n$.

Triangular Bézier patches of degree $n$ (cfr. for instance [15] for a review) are surfaces parametrised by

$$
b(u, v, w)=\sum_{i+j+k=n} \frac{n !}{i ! j ! k !} u^{i} v^{j} w^{k} b_{i j k}, \quad u+v+w=1, \quad 0 \leq u, v, w \leq 1,
$$

for a control net $\left\{b_{i j k}: i+j+k=n, 0 \leq i, j, k \leq n\right\}$ of $(n+2)(n+1) / 2$ vertices.

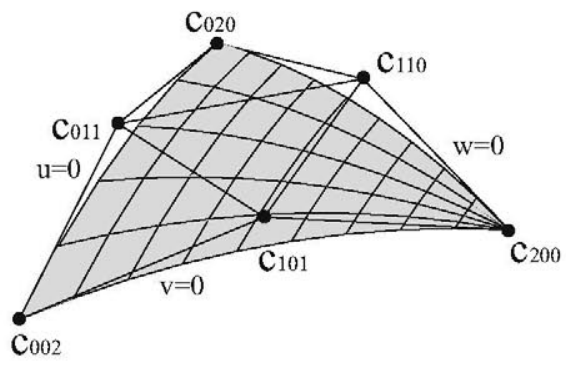

Fig. 1. Bézier triangle of degree two

The surface patch is bounded by three curves of degree $n$ (see Fig. 1) located at $u=0, v=0, w=0$ and their respective control polygons are given by $\left\{b_{0 j n-j}: j=0, \ldots, n\right\},\left\{b_{i 0 n-i}: i=0, \ldots, n\right\},\left\{b_{i n-i 0}: i=0, \ldots, n\right\}$. 
We are interested in triangular patches of ruled surfaces interpolating linearly between two curves of degree $n$ parametrised by $c(u)$ and $d(v), u, v \in[0,1]$, intersecting at $c(0)=d(0)$, with control polygons $\left\{c_{0}, \ldots, c_{n}\right\}$ and $\left\{d_{0}, \ldots, d_{n}\right\}$, so that

$$
b(u, 0,1-u)=c(u), \quad b(0, v, 1-v)=d(v) .
$$

The boundary of the patch is formed then by both curves and a straight segment at $w=0$ linking the ending points of the curves, $c_{n}$ and $d_{n}$. Obviously they have to share the other end, $c_{0}=d_{0}$.

Hence we already know the outer lines of the control net,

$$
b_{i 0 n-i}=c_{i}, i=0, \ldots, n, \quad b_{0 j n-j}=d_{j}, j=0, \ldots, n,
$$

and by the linear precision property,

$$
b_{i n-i 0}=\frac{i}{n} c_{n}+\frac{n-i}{n} d_{n}, i=0, \ldots, n,
$$

so that $b(1-v, v, 0)=(1-v) c_{n}+v d_{n}$ traces a straight segment. Hence, we have to prescribe just the inner vertices of the control net.

Since the surface is ruled we require that constant $w=W$ lines on the surface must be straight lines. In order to simplify the analysis, we extend the patch from $u+v+w=1$ to $u+v=1$, so that these lines are parametrised as

$$
\begin{aligned}
r_{W}(u) & =b(u, 1-u, W)=\sum_{k=0}^{n}\left(\begin{array}{l}
n \\
k
\end{array}\right) W^{k} r_{k}(u), \\
r_{k}(u) & :=\sum_{i=0}^{N_{k}}\left(\begin{array}{c}
N_{k} \\
i
\end{array}\right) u^{i}(1-u)^{N_{k}-i} b_{i j k},
\end{aligned}
$$

denoting $N_{k}:=n-k$

Since $\left\{1, W, \cdots, W^{n}\right\}$ are linearly independent polynomials, if $r_{W}(u)$ is to be the affine parametrization of a straight segment for all values of $W$, every $r_{k}(u)$ must be the affine parametrization of a straight segment. We consider just the case of general values of the vertices $b_{i j k}$. It is clear that, as it happens for tensor product patches [16], for special positions of the vertices other solutions could be feasible. But we are interested just in the general case.

Hence, by the linear precision property, for eack $k$, the vertices

$$
\left\{d_{n-k}=b_{0 n-k k}, b_{1 n-k-1 k}, \cdots, b_{n-k-11 k}, b_{n-k 0 k}=c_{n-k}\right\}
$$

must be equally spaced in order to have linear parametrizations of segments,

$$
b_{i n-k-i k}=\frac{i}{n-k} c_{n-k}+\frac{n-k-i}{n-k} d_{n-k}, i=0, \ldots, n-k,
$$

as we checked already for $k=0$. 
Proposition 1. A Bézier triangular patch of degree $n$ parametrised as $b(u, v, w)$ and bounded by two curves $c(u), d(v)$ of degree $n$ intersecting at $c(0)=d(0)$, with control polygons $\left\{c_{0}, \ldots, c_{n}\right\}$ and $\left\{d_{0}, \ldots, d_{n}\right\}$, so that $b(u, 0,1-u)=c(u)$ and $b(0, v, 1-v)=d(v)$ is a ruled surface if its control net is given by

$$
b_{i j k}=\frac{i c_{i+j}+j d_{i+j}}{i+j}, i+j+k=n, \quad b_{00 n}=c_{0}=d_{0} .
$$

That is, the diagonal lines of the control net are formed by points which are equally spaced between vertices of the curves with the same index. An example may be seen in Fig. 2.

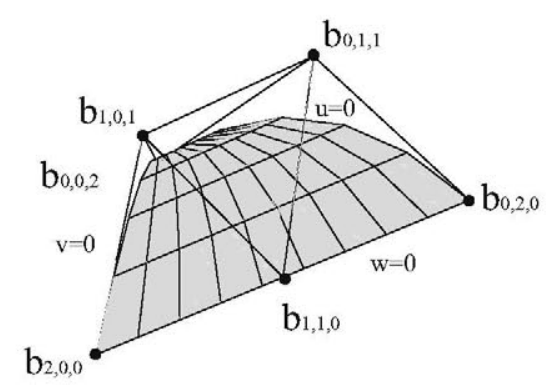

Fig. 2. Ruled Bézier triangle of degree two

For instance, for a triangle of degree four we get a control net

$$
\begin{array}{cccc}
c_{0}=d_{0} & d_{1} & d_{2} & d_{3} \\
c_{1} & \frac{c_{2}+d_{2}}{2} & \frac{c_{3}+2 d_{3}}{3} & \frac{c_{4}+3 d_{4}}{4} \\
c_{2} & \frac{2 c_{3}+d_{3}}{3} & \frac{c_{4}+d_{4}}{2} & \\
c_{3} & \frac{3 c_{4}+d_{4}}{4} & \\
c_{4} & &
\end{array} .
$$

As a counterexample, let us consider a Bézier triangle of degree two, bounded by two curves, which provide every vertex of the control net but $b_{110}$. If we choose this point aligned with $c_{2}$ and $d_{2}$, but not in the middle of the segment, it is easy to check that constant $w$ lines are not straight.

Triangular ruled patches may be related to usual explicit ruled parametrizations of surfaces,

$$
B(U, V)=(1-V) c(U)+V d(V), \quad U, V \in[0,1],
$$

by a change of coordinates,

$$
\left.\begin{array}{l}
u=U(1-V) \\
v=U V \\
w=1-U
\end{array}\right\} \Rightarrow\left\{\begin{array}{l}
U=1-w=u+v \\
V=\frac{v}{1-w}=\frac{v}{u+v}
\end{array}\right.
$$


which allow us to write down the parametrization of the ruled triangular patch in terms of the parametrizations of the curves,

$$
b(u, v, 1-u-v)=\frac{u c(u+v)+v d(u+v)}{u+v} .
$$

\section{Developable Surfaces}

Developable surfaces are ruled surfaces with null gaussian curvature $[1,2]$. Gaussian curvature, $K$, of a surface parametrised by $b(u, v)$ with unitary normal vector $\nu=b_{u} \times b_{v} /\left\|b_{u} \times b_{v}\right\|$ is defined as the quotient of the determinants of its second, $B$, and first, $G$, fundamental forms,

$$
G=\left(\begin{array}{ll}
b_{u} \cdot b_{u} & b_{u} \cdot b_{v} \\
b_{v} \cdot b_{u} & b_{v} \cdot b_{v}
\end{array}\right), \quad B=\left(\begin{array}{cc}
\nu \cdot b_{u u} & \nu \cdot b_{u v} \\
\nu \cdot b_{v u} & \nu \cdot b_{v v}
\end{array}\right), \quad K(u, v)=\frac{\operatorname{det} B(u, v)}{\operatorname{det} G(u, v)}
$$

but Gauss' Theorema Egregium states that K may be written in terms of the first fundamental form and its derivatives. Since the first fundamental form determines angles, lengths and areas on the surface, gaussian curvature is invariant under transformations, isometries, which preserve such features.

Starting with the usual parametrization of a ruled surface bounded by two curves $c(u), d(u)$,

$$
b(u, v)=(1-v) c(u)+v d(u), \quad u, v \in[0,1],
$$

since the second derivative $b_{v v}(u, v)$ is null, the determinant of the second fundamental form is negative and hence the gaussian curvature is negative or null at every point of a ruled surface. The determinant of the first fundamental form is positive, since this form is just the inner product of $\mathbb{R}^{3}$ restricted to tangent vectors to the surface.

Hence, developable surfaces are characterised by vanishing $\nu \cdot b_{u v}$ at every point, that is,

$$
\begin{aligned}
0 & =\left(d^{\prime}(u)-c^{\prime}(u)\right) \cdot\left((1-v) c^{\prime}(u)+v d^{\prime}(u)\right) \times(d(u)-c(u)) \\
& =d^{\prime}(u) \cdot c^{\prime}(u) \times(d(u)-c(u)) .
\end{aligned}
$$

This provides a useful and geometrical characterization of developable surfaces:

Proposition 2. A ruled surface parametrised as (1) is developable if and only if the vector $\mathbf{v}(u)=d(u)-c(u)$, linking the points $d(u), c(u)$, and the velocities of the curves at these points are coplanary for every value of $u$.

Or put in another way, the tangent plane is the same for all points along the straight line (generatrix or ruling of the surface) linking $d(u)$ with $c(u)$.

This means that we may write one of those velocities as a linear combination of the other two vectors,

$$
c^{\prime}(u)=\lambda(u) \mathbf{v}(u)+\mu(u) \mathbf{v}^{\prime}(u)
$$

This is useful for classifying developable surfaces: 
1. Planar surfaces: Pieces of planes are the trivial case of surfaces of null curvature.

2. Cylindrical surfaces: Ruled surfaces in which all straight lines (rulings) are parallel. For them $\mathbf{v}(u)$ is parallel to $\mathbf{v}^{\prime}(u)$.

3. Conical surfaces: Ruled surfaces in which all rulings meet at a point named vertex.

4. Tangent surfaces: Ruled surfaces formed by all tangent lines to a given curve.

The latter is the most general case, since every non-cylindrical surface may be shown to be either a tangent surface to a curve or, fulfilling additional conditions, a conical surface:

Let us perform a change of base curve from $c(u)$ by gliding it along the rulings to $\tilde{c}(u)=c(u)-\mu(u) \mathbf{v}(u)$,

$$
\tilde{c}^{\prime}(u)=c^{\prime}(u)-\mu^{\prime}(u) \mathbf{v}(u)-\mu(u) \mathbf{v}^{\prime}(u)=\left(\lambda(u)-\mu^{\prime}(u)\right) \mathbf{v}(u) .
$$

In the general case, the velocity $\tilde{c}^{\prime}(u)$ is parallel to the rulings of vector $\mathbf{v}(u)$, that is, the surface is a tangent surface to the curve $\tilde{c}(u)$. Only in the restrictive case for which $\lambda(u)=\mu^{\prime}(u), \tilde{c}^{\prime}(u) \equiv 0$, the new base curve reduces to a point, the vertex of a cone.

\section{Tensor Product Developable Patches}

In order to describe Bézier developable surfaces we start by considering a ruled surface interpolated between two polynomial curves of degree $n, c(u), d(u)$, defined by their respective control polygons, $\left\{c_{0}, \ldots, c_{n}\right\},\left\{d_{0}, \ldots, d_{n}\right\}$,

$$
c(u)=\sum_{i=0}^{n} c_{i} B_{i}^{n}(u), \quad d(u)=\sum_{i=0}^{n} d_{i} B_{i}^{n}(u),
$$

in terms of the Bernstein polynomials of degree $n$, or the de Casteljau algorithm [17],

$$
\begin{aligned}
c_{i}^{1)}(u) & =(1-u) c_{i}(u)+u c_{i+1}(u), i=0, \ldots, n-1, \\
c_{i}^{r)}(u) & =(1-u) c_{i}^{r-1)}(u)+u c_{i+1}^{r-1)}(u) i=0, \ldots, n-r, \\
c(u) & :=c_{0}^{n)}(u)=(1-u) c_{0}^{n-1)}(u)+u c_{1}^{n-1)}(u) .
\end{aligned}
$$

The derivative of the curves,

$$
c^{\prime}(u)=n\left(c_{1}^{n-1)}(u)-c_{0}^{n-1)}(u)\right), \quad d^{\prime}(u)=n\left(d_{1}^{n-1)}(u)-d_{0}^{n-1)}(u)\right),
$$

may be written as a difference between the two last-but-one points in the de Casteljau algorithm.

Hence the vectors $c^{\prime}(u), d^{\prime}(u), d(u)-c(u)$ are barycentric combinations of the points $c_{0}^{n-1)}(u), c_{1}^{n-1)}(u), d_{0}^{n-1)}(u), d_{1}^{n-1)}(u)$. Since we have already seen that the ruled surface is developable if and only if these vectors are coplanary, the developability condition for a Bézier ruled surface may be restated in terms of these: 
Proposition 3. The ruled surface interpolating between two Bézier curves of degree $n$, defined by their respective control polygons, $\left\{c_{0}, \ldots, c_{n}\right\},\left\{d_{0}, \ldots, d_{n}\right\}$ is developable if and only if the points $c_{0}^{n-1)}(u), c_{1}^{n-1)}(u), d_{0}^{n-1)}(u), d_{1}^{n-1)}(u)$ are coplanary.

That is, there exist coefficients $\Lambda(u), M(u)$, such that

$$
(1-\Lambda(u)) c_{0}^{n-1)}(u)+\Lambda(u) c_{1}^{n-1)}(u)=(1-M(u)) d_{0}^{n-1)}(u)+M(u) d_{1}^{n-1)}(u) .
$$

This way of writing the linear combination excludes the conical case. However, it does not hinder our goal of coping with the generic case.

We may gain insight into this result by rewriting it in terms of blossoms,

$$
\begin{aligned}
c_{i}^{1)}\left[u_{1}\right] & :=c_{i}^{1)}\left(u_{1}\right)=\left(1-u_{1}\right) c_{i}+u_{1} c_{i+1}, \quad i=0, \ldots, n-1, \\
c_{i}^{r)}\left[u_{1}, \ldots, u_{r}\right] & :=\left(1-u_{r}\right) c_{i}^{r-1)}\left[u_{1}, \ldots, u_{r-1}\right]+u_{r} c_{i+1}^{r-1)}\left[u_{1}, \ldots, u_{r-1}\right], \\
c\left[u_{1}, \ldots, u_{n}\right] & :=c_{0}^{n)}\left[u_{1}, \ldots, u_{n}\right], \quad i=0, \ldots, n-r, \quad r=1, \ldots, n,
\end{aligned}
$$

since the linear combinations of the points,

$$
c_{0}^{n-1)}(u)=c\left[u^{<n-1>}, 0\right], \quad c_{1}^{n-1)}(u)=c\left[u^{<n-1>}, 1\right],
$$

can be written in a rather compact form, taking into account that blossoms are multi-affine,

$$
c\left[u^{<n-1>}, \Lambda(u)\right]=d\left[u^{<n-1>}, M(u)\right] .
$$

We have therefore characterised developability of a rational ruled surface in terms of blossoms:

Theorem 1. Two Bézier curves $c(u), d(u)$ with control polygons $\left\{c_{0}, \ldots, c_{n}\right\}$, $\left\{d_{0}, \ldots, d_{n}\right\}$ define a generic developable surface if and only if their respective blossoms are related by

$$
c\left[u^{<n-1>}, A(u)\right]=d\left[u^{<n-1>}, M(u)\right]
$$

The simplest case which can be analysed is the one of constant coefficients $A$, $M$

$$
c\left[u^{<n-1>}, A\right]=d\left[u^{<n-1>}, M\right],
$$

which is the family of developable surfaces found by Aumann [12], though in that paper the key issue was the use of an affine transformation between adjacent cells of the control net of the surface.

This expression states the equality of two $(n-1)$-atic forms, which is equivalent to the equality of the respective symmetric $(n-1)$-affine forms, since the correspondence between blossoms and parametrizations is one-to-one,

$$
c\left[u_{1}, \ldots, u_{n-1}, \Lambda\right]=d\left[u_{1}, \ldots, u_{n-1}, M\right]
$$


We may draw information about the control net applying it to sequences of zeros and ones, taking into account that the vertices are recovered as

$$
\begin{gathered}
c_{j}=c\left[0^{<n-j>}, 1^{<j>}\right], \\
(1-\Lambda) c_{j}+\Lambda c_{j+1}=(1-M) d_{j}+M d_{j+1}, \quad j=0, \ldots, n-1,
\end{gathered}
$$

stating that the cells of the control net of the surface are planar and share the same linear combination between vertices.

These conditions may be solved recursively,

$$
d_{n}=\left(\frac{M-1}{M}\right)^{n} d_{0}+\frac{1-\Lambda}{M}\left(\frac{M-1}{M}\right)^{n-1} c_{0}+\frac{M-\Lambda}{M^{2}} \sum_{i=1}^{n-1}\left(\frac{M-1}{M}\right)^{n-i-1} c_{i}+\frac{\Lambda}{M} c_{n},
$$

in order to relate the first and last rulings of the patch with the vertices of the control polygon of the curve $c(u)$,

$$
\begin{aligned}
d_{n}-c_{n} & =\frac{M-\Lambda}{M}\left(\left(\frac{M-1}{M}\right)^{n-1} c_{0}+\frac{1}{M} \sum_{i=1}^{n-1}\left(\frac{M-1}{M}\right)^{n-i-1} c_{i}-c_{n}\right) \\
& +\left(\frac{M-1}{M}\right)^{n}\left(d_{0}-c_{0}\right),
\end{aligned}
$$

or even its sides,

$$
d_{n}-c_{n}=\left(\frac{M-1}{M}\right)^{n}\left(d_{0}-c_{0}\right)+\frac{\Lambda-M}{M} \sum_{i=0}^{n-1}\left(\frac{M-1}{M}\right)^{n-i-1} \Delta c_{i},
$$

denoting $\Delta c_{i}=c_{i+1}-c_{i}$.

This construction of developable Bézier surfaces can be used to solve an interpolation problem [12]:

"Given a Bézier curve $c(u)$ of degree $n$ and two straight lines $l_{0}$ and $l_{1}$ passing through the endpoints of $c(u)$, find a developable surface $b(u, v)$ through $c(u)$ $(b(u, 0)=c(u))$ with $l_{0}$ and $l_{1}$ as first and last ruling $\left(l_{0}: c(0, v), l_{1}: c(1, v)\right) . "$

Depending on the position of the rulings $l_{0}, l_{1}$ we have three possible solutions to this problem:

- If $l_{0}, l_{1}$ are parallel, we may construct a cylinder through the curve $c(u)$ with rulings parallel to $l_{0}$ and $l_{1}$.

- If $l_{0}, l_{1}$ meet at one point $V$, we may construct a cone through $c(u)$ and vertex at $V$.

- If $l_{0}, l_{1}$ are neither parallel nor meeting at one point, we may resort to Aumann's construction (8),

$$
\begin{aligned}
d_{n}-c_{n} & =\frac{A-M}{M}\left(c_{n}-a(M)\right)+\left(\frac{M-1}{M}\right)^{n}\left(d_{0}-c_{0}\right) \\
a(M) & :=\left(\frac{M-1}{M}\right)^{n-1} c_{0}+\frac{1}{M} \sum_{i=1}^{n-1}\left(\frac{M-1}{M}\right)^{n-i-1} c_{i},
\end{aligned}
$$


relating a vector on $l_{0}, d_{0}-c_{0}=\sigma \mathbf{v}$, and a vector on $l_{1}, d_{n}-c_{n}=\tau \mathbf{w}$ with a vector which is a barycentric combination of the vertices of the control polygon of the curve $c(u), c_{n}-a(M)$.

This imposes a restriction on the value of $M$ through an equation of degree $n-1$,

$$
\operatorname{det}\left(d_{0}-c_{0}, d_{n}-c_{n}, a(M)-c_{n}\right)=0 .
$$

If $M_{0}$ is a solution of this equation, we may reckon the coefficients of the linear combination,

$$
a\left(M_{0}\right)=c_{n}+\alpha_{0} \mathbf{v}+\beta_{0} \mathbf{w},
$$

solving the linear system using Cramer's rule,

$$
\alpha_{0}=\frac{\operatorname{det}\left(a\left(M_{0}\right)-c_{n}, \mathbf{w}, \mathbf{N}\right)}{\operatorname{det}(\mathbf{v}, \mathbf{w}, \mathbf{N})}, \quad \beta_{0}=\frac{\operatorname{det}\left(\mathbf{v}, a\left(M_{0}\right)-c_{n}, \mathbf{N}\right)}{\operatorname{det}(\mathbf{v}, \mathbf{w}, \mathbf{N})},
$$

where $\mathbf{N}=\mathbf{v} \times \mathbf{w}$ is a vector that completes a linear basis $\{\mathbf{v}, \mathbf{w}, \mathbf{N}\}$.

Hence, equation (10) is written as

$$
\tau_{0} \mathbf{w}=\frac{M_{0}-A}{M_{0}}\left(\alpha_{0} \mathbf{v}+\beta_{0} \mathbf{w}\right)+\left(\frac{M_{0}-1}{M_{0}}\right)^{n} \sigma_{0} \mathbf{v},
$$

from which we may read the coefficients $\sigma_{0}$ and $\tau_{0}$ that determine the ends of the rulings,

$$
\sigma_{0}=\frac{A-M_{0}}{M_{0}-1}\left(\frac{M_{0}}{M_{0}-1}\right)^{n-1} \alpha_{0}, \quad \tau_{0}=\frac{M_{0}-A}{M_{0}} \beta_{0} .
$$

The coefficient $A$ remains a free parameter and may be fixed by choosing either $d_{0}$ along $l_{0}$ or $c_{n}$ along $l_{1}$, but not both. This problem may be avoided by elevating the degree of the surface, stretching the surface patch along the rulings $d(u)-c(u)[13]$.

If we have already made use of $\Lambda$ for fixing $d_{n}$, this may be accomplished by multiplying this vector by a linear factor $(1-A) u+A$, so that the new surface patch

$$
\tilde{b}(u, v)=c(u)+v(d(u)-c(u))((1-A) u+A),
$$

is bounded by the curves $c(u)$ and $\tilde{d}(u)=c(u)+((1-A) u+A)(d(u)-c(u))$ and we may use the coefficient $A$ for choosing the end of the other ruling,

$$
\tilde{d}_{0}=\tilde{d}(0)=c_{0}+A\left(d_{0}-c_{0}\right) .
$$

As we see in the next section, this construction is useful for designing developable triangular patches.

\section{Triangular Developable Patches}

We may try to use Aumann's family of developable surfaces to construct triangular developable surfaces limited by two curves of degree $n$ and control polygons $\left\{c_{0}, \ldots, c_{n}\right\},\left\{d_{0}, \ldots, d_{n}\right\}$. The first cell of the control net is restricted by 


$$
(1-\Lambda) c_{0}+\Lambda c_{1}=(1-M) d_{0}+M d_{1},
$$

but since the curves intersect at $c_{0}=d_{0}$, the three points must be aligned,

$$
d_{1}=\left(1-\frac{\Lambda}{M}\right) c_{0}+\frac{\Lambda}{M} c_{1} .
$$

This is a severe restriction, since it implies that the initial velocities of the curves must be parallel with this construction. An example may be seen in Fig. 3 .

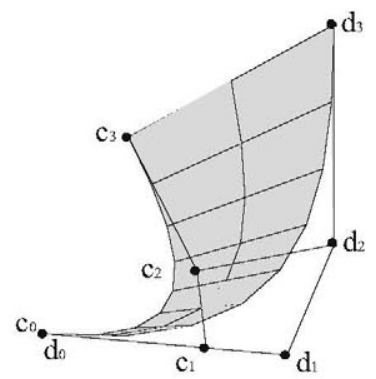

Fig. 3. Degenerate triangular developable patch

Therefore, Aumann's family of developable surfaces does not seem to be a good starting point for designing triangular patches. However, we may use them as an auxiliary patch for constructing them.

Though we do not know the direction of the ruling at the initial vertex of the triangular patch, we may use Aumann's construction to design a tensor product developable patch through a curve $c(u)$ of degree $n$ and control polygon $\left\{c_{0}, \ldots, c_{n}\right\}$ and fixing the last ruling by the choice of $d_{n}$,

$$
b(u, v)=c(u)+v \mathbf{v}(u), \quad \mathbf{v}(u)=d(u)-c(u) .
$$

We fix the unknown vertex $d_{0}$ by shortening the patch along the rulings of direction $\mathbf{v}(u)$,

$$
\tilde{b}(u, v)=c(u)+v u \mathbf{v}(u),
$$

so that the new bounding curve $\tilde{d}(u)=\tilde{b}(u, 1)$ meets $c(u)$ at $c_{0}$.

The velocity of the $v=$ const. curves is given by

$$
\frac{\partial \tilde{b}(u, v)}{\partial u}=c^{\prime}(u)+v \mathbf{v}(u)+v u \mathbf{v}^{\prime}(u) .
$$

In particular, at the beginning of the curve $\tilde{d}(u)$,

$$
\tilde{d}^{\prime}(0)=c^{\prime}(0)+\mathbf{v}(0)=n\left(c_{1}-c_{0}\right)+\left(d_{0}-c_{0}\right),
$$


we learn that we may fix the auxiliary initial ruling by prescribing the initial velocity of the bounding curve $\tilde{d}(u)$,

$$
d_{0}=(n+1) c_{0}-n c_{1}+\tilde{d}^{\prime}(0) .
$$

Hence, by this procedure it is possible to find triangular developable patches with boundary on $c(u)$ and the ruling $\overline{d_{n} c_{n}}$ fixing the value of $d^{\prime}(0)$ and making use of Aumann's construction.

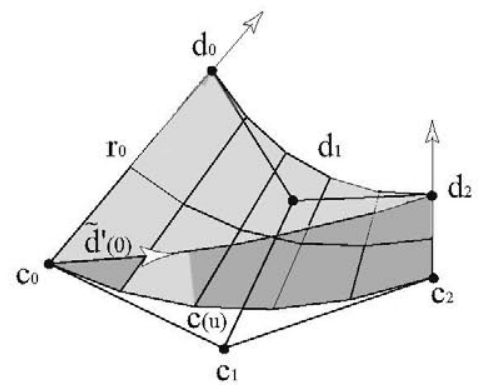

Fig. 4. Stretching a tensor product patch to a triangular patch

\section{Cylindrical and Conical Triangular Patches}

Triangular patches of cylinders and cones are easier to construct than tangent surfaces.

Cylinders bounded by a curve $c(u)$ of degree $n$ and rulings parallel to a constant vector $\mathbf{v}$ are parametrised as

$$
b(u, v)=c(u)+v f(u) \mathbf{v},
$$

where $f(u)$ is a polynomial vanishing at $u=0$. The other bounding curve is $d(u)=b(u, 1)$. An example is shown in Fig. 5 .

Hence, the only requirement for building a cylindrical triangular patch is that the vertices of the control polygons of the bounding curves, $\left\{c_{0}, \ldots, c_{n}\right\}$, $\left\{d_{0}, \ldots, d_{n}\right\}$ must lie on parallel lines,

$$
\overrightarrow{c_{1} d_{1}}\|\cdots\| \overrightarrow{c_{n} d_{n}},
$$

except for the first pair which coalesce to a single point, $c_{0}=d_{0}$.

Cones through a curve $c(u)$ and with vertex on a point $a$ may be parametrised as

$$
b(u, v)=c(u)+v \mathbf{v}(u), \quad \mathbf{v}(u)=c(u)-a .
$$

Hence, if $c(u)$ is a curve of degree $n$, a curve $d(u)$ at $v=$ const. is also of the same degree. Since such curves are scaled copies of $c(u)$, their control polygons must have sides proportional to the ones of the original curve,

$$
\overrightarrow{d_{i} d_{i-1}}=\alpha \overrightarrow{c_{i} c_{i-1}}, \quad i=1, \ldots, n,
$$




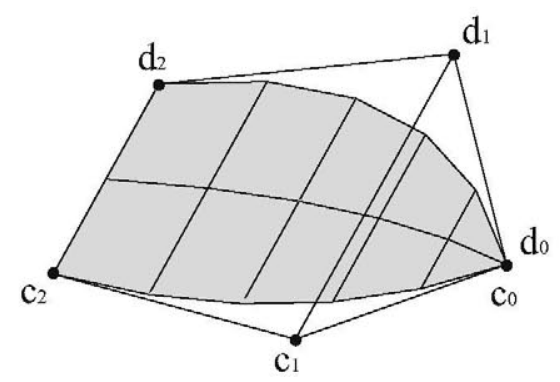

Fig. 5. Cylindrical triangular patch

being $\left\{d_{0}, \ldots, d_{n}\right\}$ the control polygon of the second curve.

We may proceed as we did for tangent surfaces in order to get triangular conical patches of degree $n+1$. We shorten the patch linearly along the ruling so that the first generatrix is reduced to a single point,

$$
\tilde{b}(u, v)=c(u)+v u \mathbf{v}(u) .
$$

The degree of the bounding curve $\tilde{d}(u)=\tilde{b}(u, 1)$ is $n+1$. An example is shown in Fig. 6.

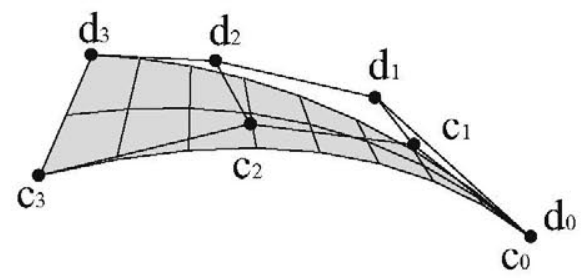

Fig. 6. Conical triangular patch

\section{Conclusions}

In this paper control nets for ruled triangular Bézier patches bounded by two curves and a straight line have been constructed. It has been shown that $\mathrm{Au}-$ mann's construction, which has been useful for designing general developable surfaces with tensor product patches, renders degenerate triangular patches. A construction grounded on degree elevation has been devised for bypassing this problem and producing nondegenerate triangular Bézier developable surfaces. This construction has been used for providing solutions to the problem of interpolating a triangular developable surface based on a curve and the last ruling of the surface, knowing the initial velocity of the other bounding curve. 
Acknowledgments. The present work has been supported by Spanish Ministry of Science grant MTM2011-22886.

\section{References}

1. Postnikov, M.M.: Lectures in Geometry: Linear Algebra and Differential Geometry. "Nauka", Moscow (1979)

2. Struik, D.J.: Lectures on classical differential geometry, 2nd edn. Dover Publications Inc., New York (1988)

3. Mancewicz, M., Frey, W.: Developable surfaces: properties, representations and methods of design. Technical report, GM Research Publication GMR-7637 (1992)

4. Frey, W., Bindschadler, D.: Computer aided design of a class of developable bézier surfaces. Technical report, GM Research Publication R\&D-8057 (1993)

5. Aumann, G.: Interpolation with developable Bézier patches. Comput. Aided Geom. Design 8(5), 409-420 (1991)

6. Lang, J., Röschel, O.: Developable $(1, n)$-Bézier surfaces. Comput. Aided Geom. Design 9(4), 291-298 (1992)

7. Chalfant, J., Maekawa, T.: Design for manufacturing using b-spline developable surfaces. J. Ship Research 42(3), 207-215 (1998)

8. Bodduluri, R., Ravani, B.: Design of developable surfaces using duality between plane and point geometries. Computer Aided Design 25(10), 621-632 (1993)

9. Pottmann, H., Farin, G.: Developable rational Bézier and $B$-spline surfaces. Comput. Aided Geom. Design 12(5), 513-531 (1995)

10. Pottmann, H., Wallner, J.: Approximation algorithms for developable surfaces. Comput. Aided Geom. Design 16(6), 539-556 (1999)

11. Chu, C.H., Séquin, C.H.: Developable bézier patches: properties and design. Computer Aided Design 34(7), 511-527 (2002)

12. Aumann, G.: A simple algorithm for designing developable Bézier surfaces. Comput. Aided Geom. Design 20(8-9), 601-619 (2003); In memory of Professor J. Hoschek

13. Aumann, G.: Degree elevation and developable Bézier surfaces. Comput. Aided Geom. Design 21(7), 661-670 (2004)

14. Fernández-Jambrina, L.: B-spline control nets for developable surfaces. Comput. Aided Geom. Design 24(4), 189-199 (2007)

15. Farin, G.: Triangular bernstein-bézier patches. Comput. Aided Geom. Design 3(2), 83-127 (1986)

16. Juhász, I., Róth, Á.: Bézier surfaces with linear isoparametric lines. Comput. Aided Geom. Design 25(6), 385-396 (2008)

17. Farin, G.: Curves and surfaces for CAGD: a practical guide. Morgan Kaufmann Publishers Inc., San Francisco (2002) 